\title{
New approvals, genetic testing, maintenance therapy, and DFS in ovarian cancer
}

\author{
Bernard A Mason, MD
}

\section{Genetic testing in women with ovarian cancer $^{1}$}

Recent study findings have indicated that women with ovarian cancer may have BRCA1 or BRCA2 mutations despite a negative family history, and current NCCN (National Comprehensive Cancer Network) guidelines endorse genetic testing for all women with epithelial cancer of the ovary. Despite this, recent reports indicate that most women with ovarian cancer are not being tested, particularly those who are elderly or without a family history. In this paper by Daniels and colleagues, the investigators examined targeted versus universal genetic testing to see if the use of a well-regarded risk model (BRCAPRO) based on personal and family history could discriminate among patients with high-grade serous ovarian cancer. Targeted genetic testing in this group might help lower costs and encourage testing for those women who actually have a significant chance of carrying a deleterious gene mutation.

In all, 589 patients with ovarian cancer seen at 3 comprehensive cancer centers were referred for genetic counseling and testing. The results showed that:

a) Women with BRCAPRO scores of less than $10 \%$ had virtually no mutations and could be spared the anxiety and expense of genetic testing.

b) Fewer mutations were observed than expected.

c) There was no difference in predictability for the BRCAPRO between high-grade and not highgrade serous tumors.

d) BRCA1/BRCA2 genetic testing should be done for all patients with high-grade serous ovarian cancer regardless of family history.

Key points

A significant percentage of women with high-grade serous ovarian cancers have BRCA1 and BRCA2 mutations. The presence of a mutation in an affected patient has important therapeutic implications as well as potential major health implications for the patient's family members. Appropriate genetic testing of family members of a patient with a BRCA mutation can be an effective strategy for prevention of future cancer or, at least, for early diagnosis.

Because genetic counseling and testing is expensive and has significant psychosocial issues associated with it, targeted rather than universal testing of ovarian cancer patients would be preferable. BRCAPRO is a widely used risk model tool based on personal and family history that might allow for such targeted testing. Unfortunately, in this study of a group of women with high-grade serous adenocarcinoma of the ovary, there were 51 mutations (28\% of all mutations) in patients with low risk scores $(<10 \%)$, which would have been missed if testing had not been done. Overall, there more mutations than expected in this population. BRCAPRO underestimated the risk for women with high-grade serous tumors, but overestimated the risk for women with other histologies. The conclusion of the paper was that all women with ovarian cancer should be tested for BRCA1 and BRCA2 mutations as per the current NCCN guidelines. Targeted testing based on personal and family history is not acceptable, particularly for those with high-grade serous tumors.

Answer, d

\section{Bevacizumab plus chemotherapy in platinum-resistant recurrent epithelial ovarian, fallopian tube, or primary peritoneal cancer $^{2-4}$}

In November 2014, the US Food and Drug Administration (FDA) approved bevacizumab in combination with paclitaxel, liposomal doxorubicin, or topotecan for the treatment of platinum-resistant

JCSO 2015;13:122-124. O2015 Frontline Medical Communications. DOI 10.12788/jcso.0120. 
ovarian cancer based on the AURELIA trial results.

\section{The following statements about the AURELIA trial are not true:}

a) It showed an improvement in disease-free survival (DFS).

b) It showed an improvement in overall survival (OS).

c) It showed a significant improvement in patient-reported outcomes.

d) It is the only phase 3 randomized trial of bevacizumab in platinum resistant ovarian cancer.

\section{Key points}

There have been 3 previous randomized phase 3 trials of bevacizumab with chemotherapy for ovarian cancer. The GOG 128 and ICON7 studies were of the combination chemotherapy followed by bevacizumab maintenance therapy in the setting of newly diagnosed ovarian cancer, and the OCEANS trial examined the combination of bevacizumab with carboplatin and gemcitabine in platinum-sensitive recurrent disease.

In the recently published AURELIA trial, patients must have had no more than 2 previous chemotherapy regimens to be eligible and had measurable or assessable progression within 6 months of completing a platinum-based regimen. All 4 bevacizumab studies showed a statistical improvement in progression-free survival (PFS), but not in OS. In the AURELIA trial, there was an absolute 3.3-month improvement, from 3.4 to 6.7 months. The AURELIA trial was unique in evaluating bevacizumab and chemotherapy in women with platinum-resistant recurrent ovarian cancer, and in being able to demonstrate an improvement of $\geq 15 \%$ in patient-related outcomes, namely abdominal and gastrointestinal symptomatology, which was statistically significant $(P=.002)$

\section{Answer, b}

\section{Pazopanib maintenance therapy in ovarian cancer $^{5-6}$}

This large randomized, phase 3 trial, is the fifth such study of an anti-angiogenic agent in the treatment of ovarian cancer, but this time the agent was the oral tyrosine kinase inhibitor, pazopanib, rather than the intravenous monoclonal antibody, bevacizumab. For the study, 940 patients with ovarian cancer who had completed primary treatment with surgery followed by at least 5 cycles of chemotherapy were randomized to receive either pazopanib or placebo.

\section{The study results included:}

a) Remarkably low toxicity.

b) An improvement in OS. c) Significant improvement in PFS.

d) East Asian patients did particularly well.

\section{Key points}

In this large phase III study of maintenance therapy with an antiangiogenic agent, the design was different from previous studies in 2 ways: first, the biological agent was pazopanib rather than bevacizumab, and second, patients were not eligible for study until they had successfully completed chemotherapy and had no evidence of progressive disease. There were a significant number of grade 3 and 4 adverse events in the pazopanib group, and one-third of patients discontinued treatment with the study drug because of toxicity. Patients from East Asia had more toxicity, dose reductions, and less efficacy than non-East Asian patients. Although there was an improvement in PFS, OS was not improved. Because of the toxicity and lack of improvement in OS, the drug manufacturer has suspended plans to seek an approval for an ovarian cancer indication in Europe, and the fate of the drug in the United States for this indication is not clear.

\section{Answer, c}

\section{Olaparib in ovarian cancer $^{7-9}$}

In June 2014, the FDA's Oncologic Drugs Advisory Committee voted 11-2 against the accelerated approval of olaparib, a PARP (poly [adenosine diphosphate-ribose] polymerase) inhibitor for the treatment of ovarian cancer.

Despite the committee's recommendation, the agency approved the drug for advanced BRCA-mutated ovarian cancer in December of that year. Approval was contingent on positive results in 2 phase 3 trials of the drug in BRCAmutated ovarian cancer, which are expected to report in 2015.

Meanwhile, the phase 2 trial of olaparib maintenance therapy in women with platinum-sensitive serous ovarian cancer that led to its 2014 accelerated approval and its preplanned retrospective analysis have been published.

In the analysis of BRCA-mutated patients, olaparib treatment resulted in:

a) No difference in toxicities from the placebo arm.

b) An improvement in OS.

c) An improvement in quality of life in the treated group.

d) An improvement in PFS.

\section{Key points}

Investigators conducted a randomized, double-blind, placebo-controlled, phase 2 study to evaluate maintenance treatment with olaparib in 265 patients with platinumsensitive, recurrent, high-grade serous ovarian cancer. The original publication in 2012 of the results for all patients 
showed a statistically significant improvement of PFS (8.4 months for the olaparib group vs 4.8 months for placebotreated group), but no difference in OS. The difference in PFS was even more striking in the preplanned retrospective analysis of outcomes by BRCA status published in 2014. For the BRCA-mutation-positive patients, the PFS for the olaparib group was 11.2 months, compared with 4.3 months for the placebo-treated group. Fatigue, nausea, and anemia were worse in the olaparib group. The FDA gave provisional approval to the drug because of the marked improvement in PFS in the BRCA mutation-enriched study population.

\section{Answer, d}

\section{Prognostication in ovarian cancer ${ }^{10}$}

Conditional survival, which accounts for the changes of the risk or mortality over time for survivors, may be more accurate than the traditional DFS, which measures survival from the time of remission. In this paper, conditional DFS and DFS were calculated for 404 patients with ovarian, fallopian tube, and peritoneal carcinoma.

\section{The analysis resulted in the following conclusions (include all that apply):}

a) DFS for patients with ovarian cancer improved over time.

b) DFS improved least dramatically over time for those who were older than 65 years, with advanced disease, and poorly differentiated tumors.

c) More accurate information about recurrence from conditional DFS information allows for better informed decisions about follow-up care.

d) Conditional DFS information improves quality of life.

\section{Key points}

Most ovarian cancer patients achieve remission, but unfortunately, most are destined to relapse and die from their disease. For patients who have already survived for a period of time, conditional DFS provides prognostic information about the likelihood of surviving another 3 years. This probability improves dramatically with time, particularly for those women with initial poor prognosis (elderly, advanced disease, and poorly differentiated tumors). More accurate information about recurrence can improve the quality of life and decisions about follow-up care.

\section{Answers, a c d}

\section{End-of-life care in the elderly with ovarian cancer $^{11}$}

In this analysis derived from the linkage of SEER registry data with Medicare over a 10 -year period, nearly 7,000 elderly women who died from ovarian cancer by December 2007 were identified and their medical care evaluated

The patients' end-of-life care was examined, and the results included:

a) Hospice care did not increase during the study period.

b) Death in the hospital increased.

c) ICU admissions increased.

d) Hospitalizations and health transitions fell.

\section{Key points}

In this population-based study of women who had died from ovarian cancer, it was disappointing that aggressive care near the end of life did not decrease although there had been an increase the use of hospice services during the study period. In fact, ICU and repeated emergency room admissions and health care transitions were increased. Patients who were within 3 days of death were more likely to enroll in hospice than were outpatients, so that the decrease in the numbers of terminal admissions was accounted for by using hospice as an "add-on" service to manage death after futile aggressive hospital-based interventions.

\section{Answer, c}

\section{References}

1. Daniels MS, Babb SA, King RH, et al. Underestimation of risk of a BRCA1 or BRCA2 mutation in women with high-grade serous ovarian cancer by BRCAPRO: a multi-institution study. J Clin Oncol. 2014;32:1249-1255.

2. Liu JF, Cannistra SA. Emerging role for bevacizumab in combination with chemotherapy for patients with platinum-resistant ovarian cancer. J Clin Oncol. 2014;32:1287-1289.

3. Pujade-Lauraine E, Hilpert F, Weber B, et al. Bevacizumab combined with chemotherapy for platinum-resistant recurrent ovarian cancer: the AURELIA open-label randomized phase III trial. J Clin Oncol. 2014;32:1302-1308.

4. Stockler MR, Hilpert F, Friedlander M, et al. Patient-reported outcome results from the open-label phase III AURELIA trial evaluating bevacizumab-containing therapy for platinum-resistant ovarian cancer. J Clin Oncol. 2014;32:1309-1316.

5. du Bois A, Floquet A, Kim J-W, et al. Incorporation of pazopanib in maintenance therapy of ovarian cancer. J Clin Oncol. 2014;32:33743384.

6. Oliver KE, McGuire WP. Ovarian cancer and antiangiogenic therapy: caveat emptor. J Clin Oncol. 2014;32:3353-3356.

7. Ledermann J, Harter P, Gourley C, et al. Olaparib maintenance therapy in patients with platinum-sensitive serous ovarian cancer: a preplanned retrospective analysis of outcomes by BRCA status in a randomized phase 2 trial. Lancet Oncol. 2014;15:852-861.

8. Ledermann J, Harter P, Gourley C, et al. Olaparib maintenance therapy in patients in platinum-sensitive relapsed ovarian cancer. $\mathrm{N}$ Eng1 J Med. 2012;366:1382-1392.

9. Oza AM, Cibula D, Benzaquen AO, et al. Olaparib combined with chemotherapy for recurrent platinum-sensitive ovarian cancer: a randomised phase 2 trial. Lancet Oncol. 2015;16:87-97.

10. Kurta ML, Edwards RP, Moysich KB, et al. Prognosis and conditional disease-free survival among patients with ovarian cancer. J Clin Oncol. 2014;32:4102-4112.

11. Wright AA, Hatfield LA, Earle CC, et al. End-of-life care for older patients with ovarian cancer is intensive despite high rates of hospice use. J Clin Oncol. 2014;32:3534-3539. 